\title{
Enfermedad diarreica aguda por Giardia lamblia
}

\author{
GERARDO M. ELIZALDE G. ${ }^{1}$, NICOLÁS ÁLVARO2², GERARDO ELIZALDE B. ${ }^{3}$ \\ ${ }^{1}$ M edico-cirujano. ${ }^{2} D$ epartamento de M edicina Humana, Facultad de M edicina - UNM SM . y D epartamento \\ de Patología Clínica, Hospital Dos de Mayo. ${ }^{3}$ D epartamento de M edicina Humana (Radiología), \\ Facultad de Medicina - U NM SM. y D epartamento de Radiología, Hospital Dos de Mayo.
}

\section{RESUMEN}

OBJETIVO: Conocer la patogenicidad de la Giardia lamblia como productora de enfermedad diarreca aguda (EDA). LUGAR: Hospital Nacional Dos de M ayo, Lima, Perú. MATERIAL Y MÉTODOS: De 20116 coproscopias provenientes de igual número de pacientes, hemos estudiado 57, por presentar G. lamblia singularmente. Provenían mayormente del Departamento de Lima. En el material fecal de ellos se realizó: 1) Reacción de Thevenon en heces; 2) Coloración de azul de metileno y lugol; 3) Concentración del parásito con la técnica de centrífugo - flotación de Faust; además 4) Hemograma; 5) Enterotest, 6) Estudio radiológico contrastado (tránsito intestinal). RESU LTAD OS: L os 57 casos presentaron diarrea, anorexia, epigastralgia, náuseas y baja ponderal. Con el azul de metileno se encontró polimorfonucleares y/o monocitos en $3 \%$. En el examen fecal seriado ( 3 muestras) se halló más de 150 quistes/gramo de heces y el enterotest verificó formas vegetativas y/o quísticas del flagelado. La reacción de Thevenon fue negativa. EI estudio radiológico en 17 de 35 casos evidenció al teraciones morfológicas a nivel del segmento duodenoyeyunal. EI hemograma reveló anemia moderada en 45 de 57 casos. La sintomatología cedió al manejo médico con metronidazol (26 giardiásicos) y/o albendazol (31 giardiásicos). CON CLUSIÓN : Giardia lamblia, flagelado cosmopolita, es una de las causas productoras de la EDA en nuestra población.

Palabras clave: Giardiasis; enfermedades gastrointestinales; diarrea; zonas tropicales; ecosistema tropical.

\section{Giardia lamblia ACUTE DIARRHEA DISEASE \\ SUMMARY}

OBJETIVE: To determine the pathogenicity of Giardia lamblia in relation to acute diarrhea. SETTIN G: D os de M ayo N ational Hospital, Lima, Peru. M ATERIALS AND METHODS: From 20116 coproscopies from same number of patients, we studied 57 who presented $\mathrm{G}$. lamblia alone. They came mainly from L ima D epartment. In the fecal material we performed: 1) Stools Thevenon reaction; 2) M ethilene blue and lugol staining; 3) Parasite concentration with Faust centrifugation - flotation technique; 4) Complete blood count; 5) Enterotest; 6) Radiological intestinal contrast study (intestinal transit). RESU LTS: The 57 cases presented diarrhea, anorexia, epigastric pain, nausea, and weigth decrease. With methilene blue polymorphonuclear cells and/or monocytes were found in 3\%. Serial stool exams (3 samples) showed over 150 cysts/gram of stools and enterotest verified vegetative and/or cystic flagelate forms. Thevenon reaction was negative. Radiologic study in 17 of 35 cases showed morphologic alterations at the duodenal-jejunum segment. Hemogram revealed moderate anemia in 45 of 57 cases. Symptomatology improved with metronidazol (26 giardiasics) and/or albendazol (31 giardiasics) treatment. CON CLUSION : Cosmopolitan flagelated G iardia lamblia is one of the causes of acute diarrhea in our population. Key words: Giardiasis; gastrointestinal diseases; diarrhea; tropical zones; tropical ecosystem.

Correspondencia:

Dr. Gerardo Martín Elizalde Gómez

Calle Ave Lira ${ }^{\circ} 161$

Lima 43, Perú

E-mail: gerardo496@yahoo.com 


\section{INTRODUCCIÓN}

L a giardiasis-infección se origina por ingestión de formas quísticas del patógeno junto con los alimentos $(1,2)$. En la primera porción del intestino se produce la exquistación, librándose la forma trofozoítica ( $\left.{ }^{3}\right)$. La virulencia de G. lamblia depende de su genotipo $\left({ }^{4}\right)$. Es altamente oportunista en hombres y animales $\left(^{5}\right)$.

En el mundo, es una de las causas más importantes de enfermedad diarreica aguda (EDA), especialmente en niños y turistas $\left({ }^{6,7}\right)$. Vehiculizada por el agua y alimentos $\left({ }^{8}\right)$, es actualmente la infección más común del intestino delgado $(9,10)$ del hombre del trópico latinoamericano. Los estudios clínicos y experimentales señalan anomalías en el linfocito $B$, que permiten la suceptibilidad a adquirir la infección por este flagelado ( ${ }^{11}$ ).

Está en proyecto el estudio del genoma de G . lamblia, que dará mayor información sobre este enigmático protozooario (12). La EDA es frecuente en el medio hospitalario del trópico latinoamericano, con sintomatología plurietiológica y variadas manifestaciones clínicas. El objetivo de la presente comunicación es señalar la importancia de $G$. lamblia en la generación de $E D A$, en pacientes atendidos en el Hospital Dos de M ayo (H2M, Lima - Perú, trópico latinoamericano).

\section{MATERIAL Y MÉTODOS}

De enero 2000 a marzo 2001, el L aboratorio "Oswaldo Hercelles Monterola" del H2M, recibió material fecal de 20116 pacientes. En 1297 de ellos $(6,44 \%)$ encontramos en la coproparasitoscopia (13) G. Iamblia, en 1240 asociada a otros enteropatógenos. Se estudió los 57 restantes, por haberse encontrado singularmente $G$. Iamblia. Para ello se elaboró una ficha, en la que se registró información sobre procedencia urbano, urbano-marginal o rural, clínica, radiología, coproparasitología. Los 57 tenían edades que fluctuaban de 1 a 80 años, siendo de ambos sexos.
De los 57 giardiásicos, se practicó en 35 de ellos el examen radiológico, consistente en un tránsito intestinal con sustancia de contraste (bario-dif).

El examen coproparasitoscópico fue seriado 3 muestras-, cuyo peso en gramos se registró para el cál culo de protozoario por gramo de especimen fecal. E ste contaje se practicó a los 57, sin dañar al patógeno, con la técnica de la sedimentación lenta en copa. Con pipeta, del fondo de la copa se tomó $1 \mathrm{~mL}$ del sedimento fecal, el que se extendió en una lámina portaobjetos; en ella se procedió a la cuenta de los quistes de G. Iamblia. Para estudiar el tamizado y comprobar la presencia de al imentos no digeridos, y el sedimento fecal para descubrir gramos de almidón y gotas de grasas, se lavó 3 especímenes fecales de cada uno de los 57 pacientes, con solución salina fisiológica a $35^{\circ} \mathrm{C}$. M ientras tanto, en cada especímen fecal de los 20116 pacientes se hizo solamente el examen directo con solución salina y lugol, después de haber realizado la técnica de centrífugo flotación de Faust. Se hizo la colaboración con azul de metileno y lugol en el frotís fecal de los 1297 pacientes que presentaron G. Iamblia.

En los 57 giardiásicos se realizó el hemograma y la reacción de Thevenon. Para la terapéutica se uso metronidazol y/o albendazol. En sólo 11 de los 57 giardiásicos recurrimos al enterotest o cuerda encapsulada.

\section{RESULTADOS}

El diagnóstico de $G$. Iamblia quedó definido por el estudio coproparasitológico de 1297 portadores de este parásito, el que estuvo asociado a otros enteropatógenos en 1240 casos; 57 (24 mujeres y 33 varones) aportaron heces francamente pastosas y estaban singularmente parasitados por G. lamblia (Tabla 1).

En los 5 trimestres sucesivos, de enero 2000 a marzo 2001, se estudió la EDA por G . lamblia en 57 pacientes que buscaron asistencia médica en el $\mathrm{H} 2 \mathrm{M}$. Ellos procedían mayoritariamente 
Tabla 1. Distribución de los pacientes según género y edad.

\begin{tabular}{cccc}
\hline Edad & Varones & Mujeres & Total \\
\hline$>1$ & 7 & 3 & 10 \\
$11-20$ & 5 & 4 & 9 \\
$21-30$ & 3 & 4 & 7 \\
$31-40$ & 7 & 6 & 13 \\
$41-50$ & 1 & 4 & 5 \\
$51-60$ & 4 & 2 & 6 \\
$61-70$ & 2 & 1 & 3 \\
$<80$ & 4 & - & 4 \\
Total & $33(57,9 \%)$ & $24(42,1 \%)$ & $57(100 \%)$ \\
\hline
\end{tabular}

de distritos de Lima: San Juan de M iraflores $(30 \%)$, La Victoria (25\%), A te-Vitarte $(33 \%)$, Cercado $(10,2 \%)$ y otros $(1,8 \%)$. L a giardiasisinfección en 1297 casos se presentó en época primaveral, alcanzándose el mayor pico en noviembre y el valle en otoño. De igual manera, la giardiasis-enfermedad (57 casos) se presentó en época primaveral (Tabla 2).

En cuanto a la caracterización clínica, los 57 pacientes presentaron diarrea $(81 \%)$ en el momento del examen clínico (Tabla 3), así como anorexia $(77,2 \%)$, epigastralgia $(75,4 \%)$, náuseas $(52,6 \%)$, baja ponderal $(22,8 \%)$, escasamente se encontró halitosis $(3,5 \%)$, eructos $(5,3 \%)$, pirosis $(5,3 \%)$, hepatomegalia $(1,8 \%)$.

En ellos, las heces coloreadas con azul de metileno demostró polimorfonucleares y/o monocitos en 3\%. La población portadora de Giardia lamblia fue 1297, que representa 6, 45\% del total de enteroparasitados.

El examen fecal seriado ( 3 muestras fecales) para confirmar el diagnóstico etiológico de los 57 casos evidenció más de 150 quistes por gramo de heces. Así también, en 11 pacientes sometidos al enterotest o cuerda encapsulada para obtener directamente líquido intestinal, verificamos la presencia de formas quísticas y/o trofozooíticas de G. Iamblia. La reacción de
Thevenon practicada fue negativa en los 57 giardiásicos.

El estudio radiológico realizado en 35 de los 57 giardiásicos se basó en un tránsito intestinal con sustancia de contraste (Bario-dif). Las radiografías de 17 pacientes adultos mostraron en las primeras fases del tránsito intestinal (a los 30 y 60 minutos) las siguientes alteraciones: deformación y engrosamiento de las válvulas conniventes y aumento de secreciones que desdibujan los pliegues y causaba cierta segmentación a nivel del segmento duodeno-yeyunal (Figura 1).

El hemograma practicado en los 57 giardiásicos reveló anemia moderada en 45 $(78,9 \%)$, en base a la cuantificación de hemoglobina, hematócrito y recuento eritrocitario (Tabla 4). L a fórmula leucocitaria no presentó cambios significativos.

Tabla 2. Presentación de la giardiasis -asociada y singular- según mes del año.

\begin{tabular}{|c|c|c|c|c|}
\hline \multirow[t]{2}{*}{ Mes } & Pacientes & \multicolumn{3}{|c|}{ Giardiasis } \\
\hline & $\mathrm{n}$ & $\begin{array}{l}\text { Con otros } \\
\text { enteroparásitos }\end{array}$ & Singular & Total \\
\hline \multicolumn{5}{|l|}{ A ño 2000} \\
\hline Enero & 1211 & 79 & 02 & 81 \\
\hline Febrero & 1365 & 66 & 01 & 67 \\
\hline Marzo & 1403 & 61 & 01 & 62 \\
\hline Abril & 926 & 37 & - & 37 \\
\hline Mayo & 1164 & 41 & 01 & 42 \\
\hline Junio & 1012 & 54 & 01 & 55 \\
\hline Julio & 1035 & 39 & 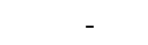 & 39 \\
\hline Agosto & 1280 & 70 & 04 & 74 \\
\hline Setiembre & 1081 & 63 & 02 & 65 \\
\hline Octubre & 1333 & 181 & 10 & 191 \\
\hline Noviembre & 1584 & 165 & 17 & 182 \\
\hline Diciembre & 1361 & 124 & 14 & 138 \\
\hline \multicolumn{5}{|l|}{ A ño 2001} \\
\hline Enero & 1934 & 91 & 01 & 92 \\
\hline Febrero & 1540 & 86 & 02 & 88 \\
\hline Marzo & 1887 & 83 & 01 & 84 \\
\hline Total & 20116 & 1240 & 57 & 1297 \\
\hline (\%) & 100 & 6,2 & 0,3 & 6,5 \\
\hline
\end{tabular}


Tabla 3. Giardiasis en 57 pacientes, según grupos etáreos, por aspectos clínicos y epi demiológicos.

\begin{tabular}{|c|c|c|c|c|c|c|c|c|c|c|c|c|}
\hline \multicolumn{2}{|c|}{ Pacientes } & \multicolumn{8}{|c|}{ Clínica } & \multicolumn{3}{|c|}{ Epidemiología } \\
\hline \multirow{2}{*}{$\begin{array}{l}\text { Edad } \\
\text { (años) }\end{array}$} & \multirow[t]{2}{*}{ № } & \multicolumn{5}{|c|}{ Sintomatología } & \multirow{2}{*}{$\begin{array}{c}\text { Signos } \\
\text { radiográficos } \\
D+E\end{array}$} & \multicolumn{2}{|c|}{ Terapéutica } & \multirow[t]{2}{*}{$\mathrm{U}$} & \multirow[t]{2}{*}{ U-M } & \multirow[t]{2}{*}{$R$} \\
\hline & & A & D & $\mathrm{E}$ & $\mathrm{N}$ & $P$ & & A & M & & & \\
\hline$>1$ & 10 & 5 & 4 & 4 & 3 & 4 & - & 8 & 2 & 3 & 7 & - \\
\hline $11-20$ & 09 & 6 & 8 & 7 & 6 & 3 & - & 4 & 5 & 2 & 7 & - \\
\hline $21-30$ & 07 & 7 & 7 & 6 & 4 & 0 & 5 & 4 & 3 & 2 & 5 & - \\
\hline $31-40$ & 13 & 11 & 10 & 10 & 6 & 1 & 9 & 5 & 8 & 2 & 9 & 2 \\
\hline $41-50$ & 05 & 5 & 5 & 4 & 2 & 2 & 2 & 5 & 0 & - & 4 & 1 \\
\hline $51-60$ & 06 & 3 & 5 & 5 & 4 & 1 & 1 & 4 & 2 & 1 & 3 & 2 \\
\hline $61-70$ & 03 & 4 & 3 & 3 & 3 & 1 & - & 0 & 3 & - & 3 & - \\
\hline$<80$ & 04 & 4 & 4 & 4 & 2 & 1 & - & 1 & 3 & 2 & 2 & - \\
\hline $\begin{array}{c}\text { TOTAL } \\
(\%)\end{array}$ & $\begin{array}{c}57 \\
(100)\end{array}$ & $\begin{array}{c}45 \\
(77,2)\end{array}$ & $\begin{array}{c}46 \\
(81)\end{array}$ & $\begin{array}{c}43 \\
(75,4)\end{array}$ & $\begin{array}{c}30 \\
(52,6)\end{array}$ & $\begin{array}{c}13 \\
(22,8)\end{array}$ & $\begin{array}{l}17 \\
(30)\end{array}$ & $\begin{array}{c}31 \\
(54,4)\end{array}$ & $\begin{array}{c}26 \\
(45,6)\end{array}$ & $\begin{array}{c}12 \\
(21,1)\end{array}$ & $\begin{array}{c}40 \\
(70,2)\end{array}$ & $\begin{array}{c}5 \\
(8,8)\end{array}$ \\
\hline
\end{tabular}

Sintomatología :

$A=$ Anorexia

$\mathrm{D}=$ Diarrea

$\mathrm{E}=$ Epigastral gia

$\mathrm{N}=$ Náuseas

$\mathrm{P}=$ Baja ponderal
Signos radiológicos:

$\mathrm{D}+\mathrm{E}=$ Dilatal ación más engrosamiento

Terapéutica : de vál vulas conniventes (I.D)

$\mathrm{A}=$ Albendazol

$M=$ Metronidazol
Epidemiología :

$\mathrm{U}=$ Urbano

$\mathrm{U}-\mathrm{M}=$ Urbano-marginal

$\mathrm{R}=$ Rural

Tabla 4. Giardi asis por grupos etáreos, según hemograma.

\begin{tabular}{|c|c|c|c|c|c|c|c|c|c|c|}
\hline \multirow[b]{2}{*}{ Hemograma } & \multicolumn{10}{|c|}{ № de paci entes (edad en años) } \\
\hline & $>1$ & $11-20$ & $21-30$ & $31-40$ & $41-50$ & $51-60$ & $61-70$ & $<80$ & Total & $\begin{array}{c}\text { Porcentaje } \\
\text { (\%) }\end{array}$ \\
\hline \multicolumn{11}{|l|}{ Hemoglobina (g/dL): } \\
\hline $7,0-9,9$ & - & - & 04 & 04 & - & 01 & - & - & 09 & 15,8 \\
\hline $10,0-12,9$ & 08 & 08 & 03 & 09 & 05 & 05 & 03 & 04 & 45 & 78,9 \\
\hline $13,0-15$ & 02 & 01 & - & - & - & - & - & - & 03 & 5,3 \\
\hline \multicolumn{11}{|l|}{ Hematócrito: } \\
\hline $25-35 \%$ & - & - & 02 & 01 & - & - & - & - & 03 & 5,3 \\
\hline $36-45 \%$ & 10 & 09 & 05 & 12 & 05 & 06 & 03 & 04 & 54 & 94,7 \\
\hline \multicolumn{11}{|l|}{ Hematíes: } \\
\hline $3,500,000-4,000,000 / \mathrm{mL}$ & - & - & 02 & 02 & - & - & - & - & 04 & 7 \\
\hline $4,000,001-4,500,000 / \mathrm{mL}$ & 09 & 10 & 05 & 11 & 05 & 06 & 03 & 04 & 52 & 91,2 \\
\hline $4,500,001-5,000,000 / \mathrm{mL}$ & 01 & - & - & - & - & - & - & - & 01 & 1,8 \\
\hline \multicolumn{11}{|l|}{ Leucocitos: } \\
\hline $5,000-10,000 / \mathrm{mL}$ & 10 & 09 & 07 & 13 & 05 & 06 & 03 & 04 & 57 & 100 \\
\hline
\end{tabular}




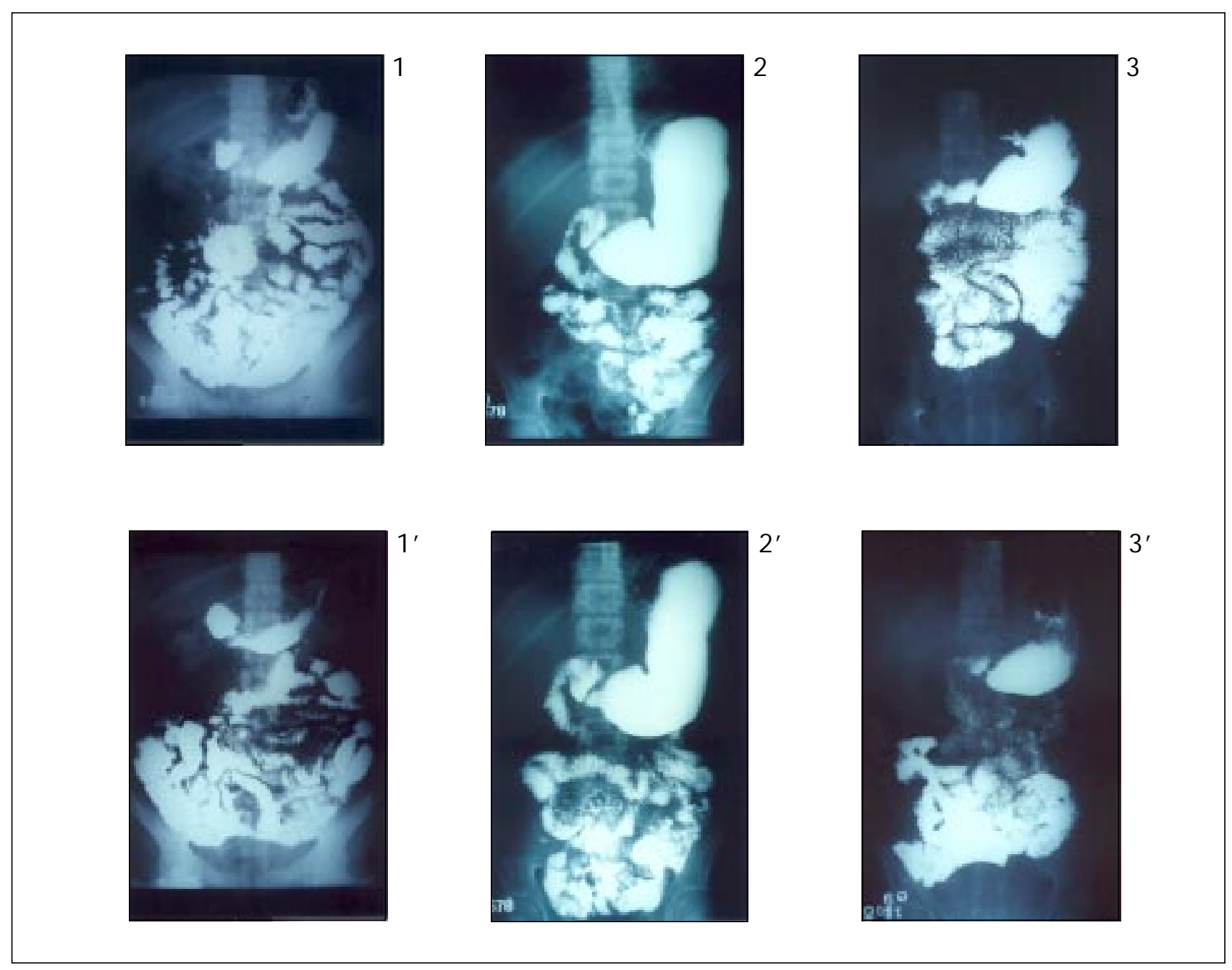

Figura 1. Se observa deformación y engrosamiento de las vál vulas conni ventes y aumento de secreciones que desdi buj a los pl iegues y causa cierta segmentación, al examen radiológico con sustancia de contraste (placas radiográficas 1, 2, 3 =a los 30 mi nutos y 1', 2', 3' =a los 60 minutos).

L a distribución de giardiasis-enfermedad (57 casos) según grupos etáreos señala mayor incidencia de 11 a 50 años de edad, logrando su mayor pico en el adulto joven y el valle en el adulto mayor. Hubo mejoría clínica con el uso de metronidazol en 26 pacientes ( 12 mujeres, 14 varones) $\mathrm{y} / 0$ albendazol en 31 pacientes ( 12 mujeres, 19 varones).

En el presente estudio, la giardiasis-enfermedad gravitó más en el medio urbano-margi- nal $(70,2 \%)$, que en el urbano $(21 \%)$ y rural $(9 \%)$.

\section{DISCUSIÓN}

Con la técnica del lavado fecal y sedimentación lenta ( ${ }^{14}$ ) en 3 especímenes fecales de cada uno de los 57 pacientes se aisló y cuantificó $G$. lamblia. En ellos no hemos investigado anticuerpos específicos $\left({ }^{5}\right)$. 
Se encontró giardiasis-infección en 1297 de 20116 personas que concurrieron al $\mathrm{H} 2 \mathrm{M}$, de enero 2000 a marzo 2001. Resultados parecidos en esa latitud de clima templado han obtenido Sullivan y Forthy en Estados Unidos de Norteamérica ${ }^{15}$ ).

Scott y col han demostrado que la colonización por este flagelado causa al teraciones difusas en el borde en cepillo del enterocito, lo cual altera la funcionalidad, originando mala absorción y mala digestión ( ${ }^{12}$ ), lo que probablemente presentaron los 1297 infectados por G. Iamblia. Guillin y col demostraron que el G. Iamblia sobrevive en el medio intestinal debido a sus proteínas de superficie $\left({ }^{16}\right)$, que le permiten sobrevida en este medio intestinal hostil $\left({ }^{17}\right)$. N uestro estudio radiológico demostró que la giardiasis-enfermedad en 17 pacientes cursó con alteraciones morfológicas a nivel de los segmentos duodeno-yeyunal, con edema y engrosamiento de la mucosa $\left({ }^{18-21}\right)$. No hemos recurrido a la intubación duodenal con estímulo de secretina (22), ni a otros antígenos que pueden obtenerse de la saliva de individuos parasitados $\left({ }^{23}\right)$. Esta protozoosis también puede ser diagnosticada recurriendo a la búsqueda de anticuerpos, con las técnicas de la inmunología ${ }^{24}$. El estado nutricional puede explicarse por la abundancia de alimento no digerido encontrado en el tamizado fecal, lo que en la clínica se traduce por baja ponderal y anemia, que encontramos en el hemograma de los 57 pacientes con giardiasis-enfermedad. Esta patología ciertamente se debe a la acción patogénica de G. Iamblia, agravado por la situación de crisis económica que caracteriza al Perú $\left({ }^{15,25}\right)$.

Además, la prevalencia de G. Iamblia productora de EDA en el trópico latinoamericano está influenciada por la calidad y cantidad de agua limpia disponible para lavar y beber $\left({ }^{26}\right)$. De 20116 pacientes que requirieron coproparasitoscopia, se encontró la giardiasisenfermedad en $0,3 \%$ y la giardiasis-infección asociada a otros enteroparásitos en $6,2 \%(27,28)$.
L as variaciones sazonales de giardiasis que verificamos en el estudio de los 57 casos, se encuentran también en reportes similares de las zonas templadas $\left({ }^{29}\right)$.

L a baja ponderal en 13 pacientes $(7,4 \%)$ se normalizó después del tratamiento médico; 10 cual ya fue señalado por Leeméca y col en modelos experimentales $\left({ }^{30,31}\right)$. No hemos investigado los niveles de inmunoglobulina $A$ sérica $y$ secretora $\left({ }^{24}\right)$, por no haber estado planificado en nuestro protocolo; sin embargo, abrimos esta posibilidad, que explicaría en cierta forma los efectos de la desnutrición en el parasitado, especialmente en los menores de 20 años.

\section{AGRADECIMIENTO}

Expresamos el agradecimiento al personal técnico del Hospital Dos de M ayo, al Departamento de Medicina Interna de la U niversidad $\mathrm{N}$ acional $\mathrm{M}$ ayor de San M arcos, así como al Departamento de Radiología de la U niversidad $\mathrm{N}$ acional $\mathrm{M}$ ayor de San M arcos, por las facilidades que nos han brindado para la realización del presente trabajo.

\section{BIBLIOGRAFÍA}

1. León Barúa R. Giardiasis. Diagnóstico 2000; 39(3): 125-6.

2. A cuña AM, Colombo H, Saúl S. Parasitosis intestinales en guarderías comunitarias de M ontevideo. Rev M ed U ruguay 1999: 5-12.

3. Vesy CJ, Peterson WL. The management of giardiasis. A liment Pharmacol Ther 1999 Jul; 13(7): 843-50.

4. Cevallos A, Forthing J. Small intestinal injury in a neonatal rat model of giardiasis is strain dependent. Gastroenterol 1995; 109: 766-73.

5. Faubert G. Immune response to Giardia duodenalis. Clin M icrobiol Reviews 2000 Jan; 13(1): 35-54.

6. Barnas G. Giardiasis in travelers. Health Care information, 17 August 2000.

7. Furness B, Beach M, Robert J. Giardiasis surveillance United States 1992-1997. CDC August 11 2000/49 (SS07) 1-13.

8. Hardie R, W all P, Gott P, Bardham M . Infectious diarrhea in tourists staying in a resort hotel. E merg Infect D is 1998. 
9. Eckmann L, Gillin F. Microbes and microbial toxins: Paradigms for microbial - mucosal interactions. Pathophysiological aspects of enteric infections with the lumen dwelling protozoan pathogen Giardia lamblia. AJP 2001 Jan; $280(1): G_{1}-G_{6}$.

10. Singer and Nash. N ormal flora in Giardia lamblia infections. J Infect Dis 2000; 181: 1510-2.

11. Scott K, Buret AG. Jejunal brush border microvillous alterations in Giardia muris-infected mice: Role of $T$ Iymphocytes and interleukin-6. Infect Immun 2000 Jun; 68(6): 3412-8.

12. Rodney D. Biology of Giardia lamblia. Clin Microbiol Reviews 2001 Jul; 14(3): 447-75.

13. G onzáles M M, L ondoño AL, Nuez FA. Control de Calidad del Diagnóstico Coproparasitológico. Centro de Investigación, Facultad de Ciencias de la Salud, U niversidad de Quindio A rmenia, marzo de 1998.

14. Faust EC, Russell PF, Yung RC. Parasitología Clínica. Barcelona:Salvat Editores SA. 1981: 59-62.

15. Sullivan $P$, Forthing $M$. Prevalence and treatment of Giardiasis in chronic diarrhea anal malnutrition. A rch Dis Childhood 1991; 66: 304-6.

16. Singer J, Elmendorf H, Conrad J, Nash T. Selection of variant - specific surface proteins in Giardia lamblia. J Infect $D$ is 2001; 183: 119-24.

17. Gillin FD, M c Caffery J M. Cell biology of the primitive eukaryote Giardia lamblia. Ann Rev M icrobiol 1996; 50: 679-705.

18. Palmer $P$, Reader $M$. The imaging of tropical diseases. $2 a$ Ed. 2000; 2: XXVI + 1684.

19. Teplick-Haskin. Diagnóstico Radiológico, 2a Edición. Interamericana, 1972: 259.

20. Laüfer I. Radiología gastrointestinal por doble contraste. Editorial M édica Panamericana SA. 1982: 426.

21. Schinz HR, Baensch WE, Frommhold W. Tratado de Roentgendiagnóstico. Tomo I Sexta Edición. Editorial Científico - M édica, 1971: 209-497.
22. Sagaro E, Castañeda C. Duodenal intubation with secretin stimulus for diagnosis of Giardiasis. A rch Dis Childhood 1977; 52: 505-7.

23. Rosales - Borjas DM , Ortiz - Ortiz L. Secretory immune response to membrane antigens during Giardia lamblia infection in humans. Infect Immunol $1998 \mathrm{Feb}$; 66(22): 756-9.

24. Torbay M. Niveles de Inmunoglobulina "A" Sérica y Secretoria en niños con Giardiasis. D epartamento de Pediatría Hospital Central Antonio María Pineda Barquisineto, 1988.

25. Saiman L, Schulte J. Prevalence of infectious diseases among internationally adopted children. Pediatrics $2001 \mathrm{Sep}$; 108(3): 608-12.

26. Steiner TS, Guerrant RL. Protozoal agent: $W$ hat are the dangers for the public water supply? A nn Rev Med 1997; 48: $329-40$.

27. Canese A, Barrios E, Castro L, Canese J. Prevalencia de parasitosis intestinales encontrados en niños en Paraguay. Rev Med M icrobiol 1999: 31-5.

28. Devera R, Niebla-Punos G, Velásquez-Alvarez $V$, Gonzáles-M eneses R. Giardiasis en escolares de Ciudad Bolívar Estado de Bolívar-Venezuela. Rev Biomed 1998; 9: 145-50.

29. Grabowski DJ, Tiggs KJ, Hall J D. Epidemiologic notes and reports common-source outbreak of Giardiasis - N ew M exico. E pidemiology Program Office CDC, J une 16 1989/ 38 (23), 403-7.

30. L eméeca V, Zahariaa I, Nevezb G, Rabodonirinac M, B rasseura $P$, Balletd J, Favenneca $L$. M etronidazole and albendazole susceptibility of 11 clinical isolates of Giardia duodenal is from F rance. J A ntimicrob Chemother 2000; 46: 819-21.

31. Harris J, Plummer S, Turner M, Lloyd D. The microaerophilic flagellate Giardia intestinalis: Allium sativum (garlic) is an effective antigiardial. M icrobiol 2000; 146: 3119-27. 